\title{
Large Starspot Groups on HAT-P-11 in Activity Cycle 1
}

\author{
Brett M. Morris, ${ }^{1}$ Suzanne L. Hawley, ${ }^{1}$ and Leslie $\mathrm{Hebb}^{2}$ \\ ${ }^{1}$ Astronomy Department, University of Washington, Seattle, WA 98195, USA \\ ${ }^{2}$ Physics Department, Hobart and William Smith Colleges, Geneva, NY 14456, USA
}

\section{BACKGROUND}

HAT-P-11 is a planet-hosting K4V star in the Kepler field, with an activity cycle that bear similarities to the Sun's (Bakos et al. 2010; Sanchis-Ojeda \& Winn 2011; Morris et al. 2017a). The chromospheric activity of HAT-P11 passed through minimum in 2016, and seems to be rising back towards maximum (Morris et al. 2017b). In late 2017, HAT-P-11's $S$-index had returned to $S \sim 0.55$, similar to the activity level in the previous cycle (Cycle 0 ). We report ground-based observations to measure the starspots of HAT-P-11 in its second observed magnetic activity cycle (hereafter Cycle 1). Spot occultations have not yet been observed at the present phase of HAT-P-11's activity cycle.

\section{OBSERVATIONS}

We gathered photometry of HAT-P-11 and six comparison stars through a holographic diffuser on the ARCTIC imager mounted on the Astrophysical Research Consortium (ARC) $3.5 \mathrm{~m}$ Telescope at Apache Point Observatory (APO) (Huehnerhoff et al. 2016). The diffuser enables precision photometry from the ground (Stefansson et al. 2017). We observed a transit of HAT-P-11 b using $2 \times 2$ binning 10 second exposures in SDSS $r$ on 2017 October 30 UTC. We normalize the HAT-P-11 light curve by a mean comparison star, which is computed from a linear combination of the following regressors: the fluxes of each comparison star, the target centroid pixel $x$ and $y$ coordinates, median sky background, air humidity, air pressure, and airmass - see Morris et al. (2018) for detailed explanation of the photometric technique. The standard deviation of the target flux beforetransit is $400 \mathrm{ppm}$ in one minute bins, $\sim 4 \times$ larger than Kepler's photometric precision (90 ppm).

\section{STARSPOT PROPERTIES}

In Figure 1, the transit light curve shows a occultation of a large dark feature in the southern hemisphere before midtransit, and a smaller dark feature in the northern hemisphere after mid-transit. We noted in Morris et al. (2017a) that adjacent spot occultations could give the appearance of much larger spots, and we emphasize here that each of these apparent spot occultations could in fact be occultations of groups of spots. If these anomalies are in fact occultations of individual spots, they would be among the largest observed on HAT-P-11. However, given the similarities between the spots observed during the Kepler years (Morris et al. 2017a) and sunspots (Solanki 2003), we suggest that we are likely observing occultations of large spot groups appearing at the beginning of Cycle 1. Spot occultations have not previously been observed at this phase of the HAT-P-11 activity cycle.

Fits with the STSP photometric spot model (Morris et al. 2017a; Hebb et al. 2018) reveal the area coverage of spots within the transit chord for UTC 2017-10-30 is 14\% — which makes this transit the most spotted HAT-P-11 transit observed to date. From 2009-2013, we measured the fraction of the transit chord occupied by spots in Morris et al. (2017a) to vary between $0.5-10 \%$.

We thank Gudmundur Stefansson and Yiting Li for productive conversations. Based on observations obtained with the APO 3.5-meter telescope, which is owned and operated by ARC.

Software: astropy (Astropy Collaboration et al. 2013), photutils (Bradley et al. 2016), friedrich (Morris et al. 2017a), astroplan (Morris et al. 2017c), STSP (Hebb et al. 2018), astroscrappy (van Dokkum et al. 2012)

Facility: APO/ARC $3.5 \mathrm{~m}$

\section{REFERENCES}




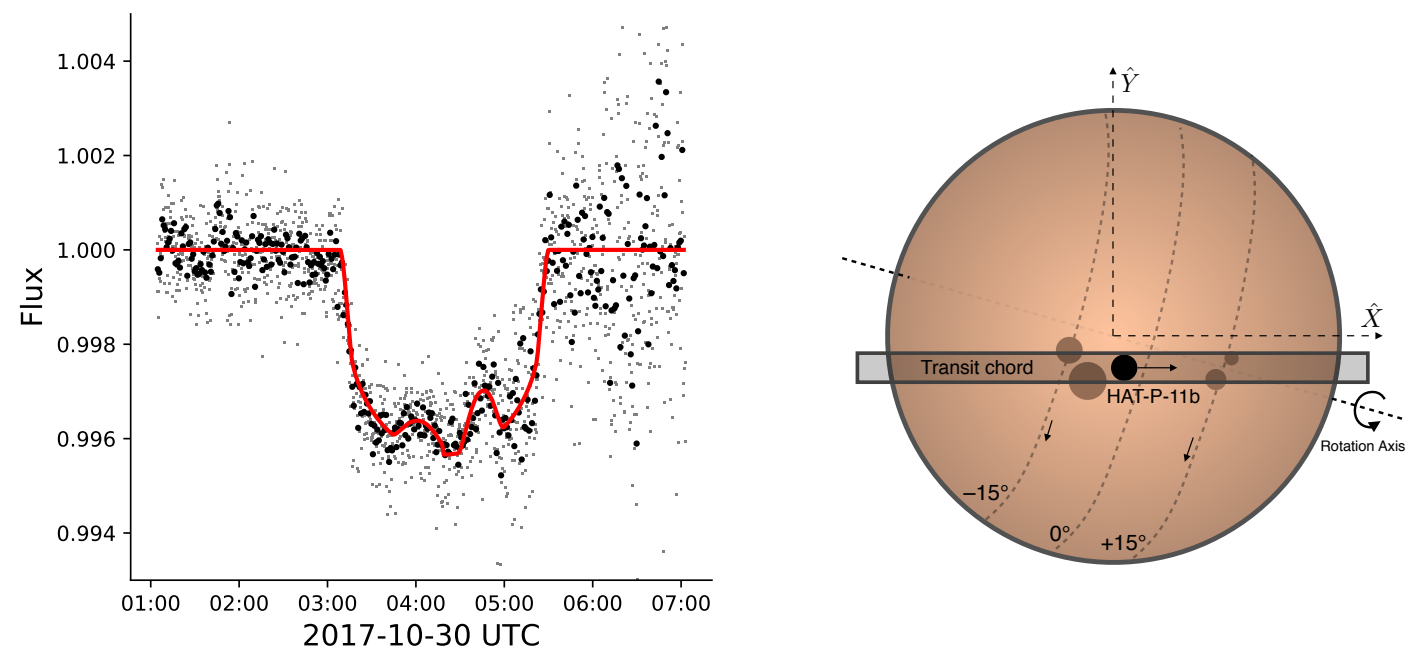

Figure 1. Left: Transit light curve at 10 second cadence (gray squares) and one minute bins (black circles), with the maximumlikelihood spot model from STSP (red curve). The scatter increases at later times due to high airmass. Right: schematic illustration of a possible spot group configuration consistent with the APO occultation photometry. See Figure 3 of Morris et al. (2017a) for a more detailed explanation of the notation.

Astropy Collaboration, Robitaille, T. P., Tollerud, E. J., et al. 2013, A\&A, 558, A33

Bakos, G. Á., Torres, G., Pál, A., et al. 2010, ApJ, 710, 1724

Bradley, L., Sipocz, B., Robitaille, T., et al. 2016,

Photutils: Photometry tools, Astrophysics Source Code

Library, , , ascl:1609.011

Hebb, L., Rohn, G., D. J. R. A. M. B. M., \& collaborators. 2018

Huehnerhoff, J., Ketzeback, W., Bradley, A., et al. 2016, in Proc. SPIE, Vol. 9908, Ground-based and Airborne Instrumentation for Astronomy VI, 99085H

Morris, B. M., Agol, E., \& Hawley, S. L. 2018, RNAAS

Morris, B. M., Hebb, L., Davenport, J. R. A., Rohn, G., \& Hawley, S. L. 2017a, ApJ, 846, 99
Morris, B. M., Hawley, S. L., Hebb, L., et al. 2017b, ApJ, 848,58

Morris, B. M., Tollerud, E., Sipocz, B., et al. 2017c, ArXiv e-prints, arXiv:1712.09631

Sanchis-Ojeda, R., \& Winn, J. N. 2011, ApJ, 743, 61

Solanki, S. K. 2003, A\&A Rv, 11, 153

Stefansson, G., Mahadevan, S., Hebb, L., et al. 2017, ApJ, 848,9

van Dokkum, P. G., Bloom, J., \& Tewes, M. 2012,

L.A.Cosmic: Laplacian Cosmic Ray Identification, Astrophysics Source Code Library, , , ascl:1207.005 